\title{
Archivos \\ de Cardiología de México

\section{Influencia de la edad en el pronóstico a corto y largo plazo del choque cardiogénico de origen isquémico}

\author{
Víctor M. Hidalgo-Olivares*, Juan Gabriel Córdoba-Soriano, Isabel López-Neira, \\ Manuel Fernández-Anguita, Cristina Llanos-Guerrero, \\ Francisco Salmerón-Martínez, Esther Cambronero-Cortinas, Carlos \\ Lafuente-Gormaz, Antonia Tercero-Martínez, Miguel Corbí-Pascual, \\ Jesús Jiménez-Mazuecos, Antonio Gutiérrez-Díez y Alfonso Valle-Muñoz
}

Servicio de Cardiología, Hospital General Universitario de Albacete, Albacete, España

Recibido el 17 de enero de 2013; aceptado el 21 de octubre de 2013

\author{
PALABRAS CLAVE \\ Choque cardiogénico; \\ Edad; \\ Pronóstico a largo \\ plazo; \\ Revascularización \\ coronaria; \\ España
}

\begin{abstract}
Resumen
Objetivo: Comparar la evolución durante el ingreso y a largo plazo de pacientes con choque cardiogénico de origen isquémico.

Método: Estudio observacional prospectivo unicéntrico llevado a cabo en la unidad coronaria de un hospital terciario manejada por cardiólogos. Se incluyen pacientes con choque cardiogénico de origen isquémico que recibieron revascularización coronaria precoz. Dividimos a los pacientes en 2 grupos: mayores de 75 años (grupo A) y menores (grupo B), y comparamos la evolución durante el ingreso y en un seguimiento máximo de 4 años. El objetivo principal fue estimar la mortalidad a los 4 años. El objetivo secundario se definió como la mortalidad intrahospitalaria. Resultados: Incluimos a 97 pacientes, 44 del grupo A (45\%). Los pacientes del grupo B eran con más frecuencia varones ( 81 vs. $57 \%, p=0.014)$, diabéticos ( 49 vs. $21 \%, p: 0.006)$ y fumadores (39.6 vs. $4.5 \%, p<0.05)$. La mortalidad hospitalaria fue superior en el grupo A $(54.5$ vs. $30.2 \%$, $\mathrm{p}=0.022$ ). El objetivo principal ocurrió en 32 pacientes del grupo A frente a 20 del grupo $B$ (73 vs. $38 \%, p=0.007)$.

Conclusión: El choque cardiogénico de origen isquémico en paciente mayores de 75 años presenta una alta mortalidad durante la estancia hospitalaria y en el seguimiento a largo plazo.

(C) 2013 Instituto Nacional de Cardiología Ignacio Chávez. Publicado por Masson Doyma México S.A. Todos los derechos reservados.
\end{abstract}

\footnotetext{
* Autor para correspondencia: C/ Hermanos Falcó 37. CP 02006 Albacete (España). Teléfono: 0034620247351.

Correo electrónico: vmhidalgo@ono.com (V.M. Hidalgo-Olivares).
} 


\section{KEYWORD}

Cardiogenic shock; Age;

Long term mortality;

Coronary revascularization;

Spain
Influence of age in short and long term prognostic of ischemic cardiogenic shock

\begin{abstract}
Objective: To compare the prognosis during hospitalization and maximum follow-up of 4 years in patients with myocardial infarction complicated with cardiogenic shock.

Method: Prospective observational study practiced in a coronary Care Unit managed by cardiologists. We included patients with myocardial infarction complicated with cardiogenic shock who received early coronary revascularization. Patients were divided into two groups: older than 75 years (group A) and lower (group B), and we compared the evolution during hospitalization and maximum follow-up of 4 years. Primary end point was mortality rate in the maximum follow-up of 4 years. Secondary end point was mortality rate during hospitalization.

Results: Ninety-seven patients were included, 45\% Group A. Patients of Group B were mostly men ( $81 \%$ vs. $57 \%$; $P=.014)$, diabetics ( $48 \%$ vs. $21 \%$; $P=0.006)$, and smokers $(39.6 \%$ vs. $5 \%)$. Mortality rate during hospitalization was higher in Group A (54.5\%) vs. $30.2 \%$ in Group B $(P=.022)$. Mortality rate during follow-up (primary variable) was $73 \%$ in Group A vs. $38 \%$ in Group B $(P=.007)$.

Conclusions: Myocardial infarction complicated with cardiogenic shock in elderly patients is an entity with high mortality during hospitalization and continues to worsen during long term follow-up.

(C) 2013 Instituto Nacional de Cardiología Ignacio Chávez. Published by Masson Doyma México S.A. All rights reserved.
\end{abstract}

\section{Introducción}

A pesar de los avances diagnósticos y terapéuticos experimentados en las últimas 4 décadas, el infarto agudo de miocardio (IAM) continúa siendo un problema importante en el mundo desarrollado y cada vez más relevante en los países en vías de desarrollo ${ }^{1,2}$. Adicionalmente, el envejecimiento acelerado de nuestra sociedad hace que cada vez haya más pacientes mayores de 75 años en las unidades coronarias, muchos de ellos octogenarios y nonagenarios. La mayor parte de los cerca de 70.000 IAM con elevación del segmento ST (IAMEST) que se producen al año en España son sufridos por pacientes mayores de 75 años $^{3}$, lo que obliga a realizar procedimientos invasivos en un grupo de población con elevado riesgo de complicaciones. A pesar de los avances en el tratamiento de revascularización en el síndrome coronario agudo, el choque cardiogénico continúa siendo la principal causa de muerte hospitalaria de los pacientes con IAM, con una incidencia que oscila entre el $5^{4}$ y el $8 \% 5,6$. El intervencionismo coronario parece mejorar el pronóstico en pacientes seleccionados ${ }^{7,8}$, aunque la evolución del choque cardiogénico en pacientes mayores de 75 años está menos estudiada. El objetivo principal de nuestro estudio es evaluar la influencia de la edad en el pronóstico a largo plazo de los pacientes con choque cardiogénico de origen isquémico manejado de manera invasiva precoz.

\section{Métodos}

Realizamos un estudio prospectivo de los pacientes que ingresaron en la Unidad Coronaria de nuestro centro entre los años 2006-2011 con diagnóstico de choque cardiogénico de origen isquémico. Todos ellos dieron consentimiento informado para su participación en el estudio, que fue aprobado por el comité de ética de nuestra institución.

\section{Criterios de inclusión}

Fueron incluidos todos los pacientes con diagnóstico de choque cardiogénico secundario a un IAM (con y sin elevación del segmento ST) a los que se les realizó revascularización coronaria percutánea en las primeras $18 \mathrm{~h}$ del inicio del cuadro clínico. Se incluyeron tanto los pacientes con choque secundario a fallo predominante de ventrículo izquierdo como los pacientes con choque secundario a fallo predominante de ventrículo derecho. La muestra fue dividida en 2 grupos: grupo A (pacientes mayores de 75 años) y grupo B (pacientes menores de 75 años), y en ambos grupo se evaluaron variables demográficas como el sexo, la presencia de factores de riesgo cardiovascular (hipertensión arterial, diabetes mellitus, antecedentes de tabaquismo), antecedentes de enfermedad arteriosclerótica previa, patologías asociadas, la anatomía coronaria con la presencia de enfermedad multivaso, el uso de balón de contrapulsación y el uso de fármacos inhibidores de la glucoproteína Ilb/IIla.

\section{Criterios de exclusión}

Fueron excluidos los pacientes con choque cardiogénico de origen isquémico a los que no se les pudo realizar intervencionismo coronario en las primeras $18 \mathrm{~h}$ del inicio del cuadro y los pacientes con choque cardiogénico de origen arrítmico. También fueron excluidos los pacientes que presentaron complicaciones mecánicas (ruptura de la pared libre ventricular, ruptura del tabique interventricular e insuficiencia mitral aguda secundaria a rotura o distensión del aparato subvalvular) o los que no dieron su consentimiento para participar en el estudio. 


\subsection{Definición de variables}

- Objetivo principal: mortalidad durante el seguimiento.

- Variable secundaria: mortalidad durante la estancia hospitalaria.

- Choque cardiogénico: coexistencia de 1) presión arterial sistólica $<90 \mathrm{mmHg}$ (o caída de $30 \mathrm{mmHg}$ sobre niveles basales) durante más de 30 min a pesar una adecuada repleción de volumen o necesidad de aminas simpaticomiméticas para mantener la presión sistólica $>90 \mathrm{mmHg}$; 2) signos de hipoperfusión tisular (oliguria $<30 \mathrm{ml} / \mathrm{h}$, frialdad y cianosis periférica u obnubilación); 3) demostración de elevación de las presiones de llenado de ventrículo izquierdo (mediante signos radiológicos compatibles con congestión pulmonar evidenciados en la radiografía de tórax, o tras demostrar mediante ecocardiografía la existencia de parámetros compatibles con aumento de presión telediastólica del ventrículo izquierdo).

- Enfermedad arteriosclerótica previa: se definió como la presencia de al menos uno de los siguientes:

- Antecedentes de cardiopatía isquémica previa.

- Antecedentes de enfermedad arterial periférica diagnosticada por el servicio de cirugía vascular.

- Patologías asociadas: se consideró la presencia de al menos una de las siguientes:

- Antecedentes de accidente cerebrovascular.

- Antecedentes de diagnóstico de EPOC por el servicio de neumología.

- Antecedentes de insuficiencia renal crónica (siendo definida esta como filtrado glomerular $<60 \mathrm{ml} / \mathrm{min}$ por $1.73 \mathrm{~m}^{2}$ durante 3 meses o más, con o sin daño renal).

- Enfermedad multivaso: se consideró enfermedad multivaso la presencia de estenosis coronaria $\geq 70 \%$ evidenciada mediante coronariografía en las 3 arterias coronarias principales (descendente anterior, circunfleja y coronaria derecha) o la presencia de estenosis $\geq 50 \%$ en el tronco coronario izquierdo.

\section{Evaluación ecocardiográfica}

A todos los pacientes se les realizó un ecocardiograma transtorácico (GE VIVID 3, EE. UU.) en el que se evaluó la fracción de eyección del ventrículo izquierdo (FEVI) por el método Simpson, definiendo como función ventricular normal la presencia de una $\mathrm{FEVI}>55 \%$, disfunción ventricular ligera FEVI 45-55\%, disfunción ventricular moderada FEVI 35 $45 \%$ y disfunción ventricular severa $\mathrm{FEVI}<35 \%$. La función sistólica de ventrículo derecho fue valorada mediante el desplazamiento sistólico del anillo lateral tricuspideo (TAPSE), definiendo disfunción sistólica de ventrículo derecho como TAPSE < $14 \mathrm{~mm}$. Los parámetros ecocardiográficos evaluados para determinar aumento de las presiones de llenado ventricular izquierdo fueron un valor del cociente $E / E^{\prime}$ medido en el anillo lateral mitral superior a 15.

\section{Estudio angiográfico}

Se realizó por médicos hemodinamistas con un equipo Philips Allura FD10 (Holanda) mediante punción arterial (radial o femoral según las características del paciente y la dificultad del procedimiento) y cateterización selectiva coronaria y realizando las proyecciones angiográficas habituales. El análisis de las imágenes fue realizado por el hemodinamista responsable del procedimiento y se determinó de forma visual la presencia de lesiones coronarias. Se consideró como significativas las lesiones que por estimación visual ocasionaban reducción de calibre $\geq 70 \%$ y en el caso del tronco coronario izquierdo $\geq 50 \%$.

\section{Análisis estadístico}

Todo el análisis estadístico se realizó mediante el paquete de software estadístico para ciencias sociales (SPSS v. 17.0 para Windows, SPSS Inc., Chicago, Illinois, EE. UU.). Mediante el test de Kolmogorov-Smirnov se ha comprobado la normalidad en la distribución en las variables cuantitativas. Todas las variables continuas y normales se expresaron como media \pm desviación estándar, el resto como porcentajes. La comparación entre grupos independientes de las variables continuas se realizó por medio de la prueba de análisis de varianza (ANOVA) con la corrección de Bonferroni para las comparaciones múltiples. La comparación entre grupos de las variables no continuas se realizó mediante el test $\chi 2$ y la prueba exacta de Fisher cuando fue apropiado. Se consideró estadísticamente significativa una probabilidad bilateral $<0.05$. La determinación de la velocidad de eventos clínicos a lo largo del seguimiento se evalúa mediante la tasa central media relativa ${ }^{9}$. En el estudio de probabilidad de supervivencia, en función de edad del paciente, se utilizó el método actuarial de Kaplan-Meier.

\section{Resultados}

\section{Características basales}

Durante los años 2006-2011 ingresaron en la Unidad Coronaria de nuestro centro un total de 97 pacientes con diagnóstico de choque cardiogénico de origen isquémico, de los cuales 44 fueron pacientes mayores de 75 años (grupo A) y 53 menores de 75 años (grupo $B$ ), con un mayor número de varones en el grupo $B$ (tabla 1 ). No existieron diferencias en los antecedentes de enfermedad ateroesclerótica previa, enfermedades asociadas, hipertensión arterial, aunque sí en el resto de los factores de riesgo. Los pacientes incluidos en el grupo $B$, los más jóvenes, tenían una mayor prevalencia de diabetes ( 49 vs. $21 \%, \mathrm{p}=0.006$ ) y de hábito tabáquico ( 39 vs. $4 \%, p=0.001$ ).

Respecto al tratamiento médico, todos los pacientes incluidos en el estudio fueron tratados de acuerdo con las guías con doble antiagregación (ácido acetilsalicílico y clopidogrel) junto a dosis altas de estatinas. Ambos grupos recibieron tratamiento con betabloqueantes e IECA según tolerancia, sin existir diferencia significativa entre ellos. Para el tratamiento del choque cardiogénico se usaron fármacos inotrópicos según las indicaciones establecidas y criterio del médico responsable del paciente.

\section{Tipo de infarto agudo de miocardio, localización y anatomía coronaria}

El origen del choque cardiogénico en la muestra de pacientes fue un IAMEST en el $82 \%$ de los pacientes y un infarto 
Tabla 1 Características basales

\begin{tabular}{|c|c|c|c|}
\hline Características basales & $\begin{array}{l}\text { Grupo A (> } 75 \text { años) } \\
\text { Media } 69 \text { años }(61-72)\end{array}$ & $\begin{array}{l}\text { Grupo B (<75 años) } \\
\text { Media } 82 \text { años }(80-85)\end{array}$ & $\mathrm{p}$ \\
\hline$n$ & $44(45 \%)$ & $53(55 \%)$ & - \\
\hline \multicolumn{4}{|l|}{ Sexo } \\
\hline Mujeres & $19(43 \%)$ & $10(19 \%)$ & 0.014 \\
\hline Hipertensión & $34(77 \%)$ & $35(66 \%)$ & 0.26 \\
\hline Diabetes mellitus & $9(21 \%)$ & $26(49 \%)$ & 0.006 \\
\hline Tabaquismo & $2(4.5 \%)$ & $21(39.6 \%)$ & 0.001 \\
\hline Patologías asociadas & $8(18.1 \%)$ & $10(18.8 \%)$ & 1 \\
\hline Enfermedad aterosclerótica previa & $9(21 \%)$ & $19(35.8 \%)$ & 0.4 \\
\hline \multicolumn{4}{|l|}{ Tipo de infarto de miocardio } \\
\hline IAMEST & $36(81 \%)$ & $44(83 \%)$ & 0.9 \\
\hline IAMSEST & $8(19 \%)$ & 9 (17\%) & \\
\hline \multicolumn{4}{|l|}{ Localización del infarto } \\
\hline Anterior/lateral & $18(41 \%)$ & $22(41.5 \%)$ & \\
\hline Inferior/posterior & $6(13.7 \%)$ & 7 (13.2\%) & $p=0.82$ \\
\hline Inferior + VD & $12(27.3 \%)$ & $15(28.3 \%)$ & \\
\hline No Q & $8(18 \%)$ & $9(17 \%)$ & \\
\hline Enfermedad multivaso & 18 (41\%) & 19 (35.8\%) & 0.67 \\
\hline FEVI & $34 \% \pm 11$ & $37 \% \pm 14$ & $p=0.84$ \\
\hline
\end{tabular}

FEVI: fracción de eyección del ventrículo izquierdo; IAMEST: infarto agudo de miocardio con elevación del segmento ST; IAMSEST: infarto agudo de miocardio sin elevación del segmento ST; VD: ventrículo derecho.

Los valores se expresan como $\mathrm{n}(\%)$.

agudo de miocardio sin elevación del segmento ST en el $18 \%$ restante, sin que existiesen diferencias significativas respecto a la incidencia del tipo de síndrome coronario agudo entre ambos grupos (36 pacientes [81\%] IAMEST grupo A y 44 pacientes [83\%] en el grupo $B, p=0.9$ ) o en cuanto a la localización del IAM (41\% localización anterior/lateral en el grupo $A$ y $41.5 \%$ en el grupo $B, p=0.82$ ). Tampoco se hallaron diferencias en cuanto al resto de las localizaciones. La enfermedad multivaso se objetivó en el $41 \%$ de los pacientes del grupo A y en el $35 \%$ del $B(p=0.67)$.

\section{Hallazgos ecocardiográficos}

La ecocardiografía realizada en las primeras $24 \mathrm{~h}$ del ingreso mostró una FEVI en los pacientes del grupo A del $34 \pm 11 \%$ y en los pacientes más jóvenes del $37 \pm 14 \%$ en el grupo $B$ $(p=0.8)$. En cuanto a los parámetros de disfunción diastólica del ventrículo izquierdo y de disfunción sistólica del ventrículo derecho, no se presentaron diferencias significativas entre ambos grupos.

\section{Revascularización multivaso, uso de anti-Ilb/Illa y balón intraaórtico de contrapulsación}

Se realizó revascularización coronaria percutánea a todos los pacientes incluidos en el estudio en las primeras $18 \mathrm{~h}$ del desarrollo del cuadro, implantándose stent convencional o recubierto según la preferencia del hemodinamista, sobre la arteria responsable del SCA, exceptuando en el $20.45 \%$ de los pacientes del grupo A y en el $15 \%$ de los pacientes del grupo $B(p=0.59)$, en los que se realizó revascularización sobre 2 o más vasos. El uso de fármacos inhibidores de la glucoproteína Ilb/Illa fue similar en ambos grupos $\mathbf{3 1 . 8 \%}$ pacientes del grupo A vs. $41.5 \%$ de pacientes del grupo $B, p=0.4)$; de la misma manera, el implante pre o postintervencionismo del balón de contrapulsación intraaórtico no presentó diferencias entre ambos grupos de la muestra (31.8\% grupo A vs. $41.5 \%$ grupo $B, p=0.4$ ) (fig. 1).

\section{Análisis de supervivencia}

Durante el periodo de seguimiento máximo de 4 años, no hubo pérdida de seguimiento de ningún paciente (todos los pacientes incluidos fueron evaluados) y fallecieron 52 pacientes del total de la muestra $(53.6 \%)$. La mediana de

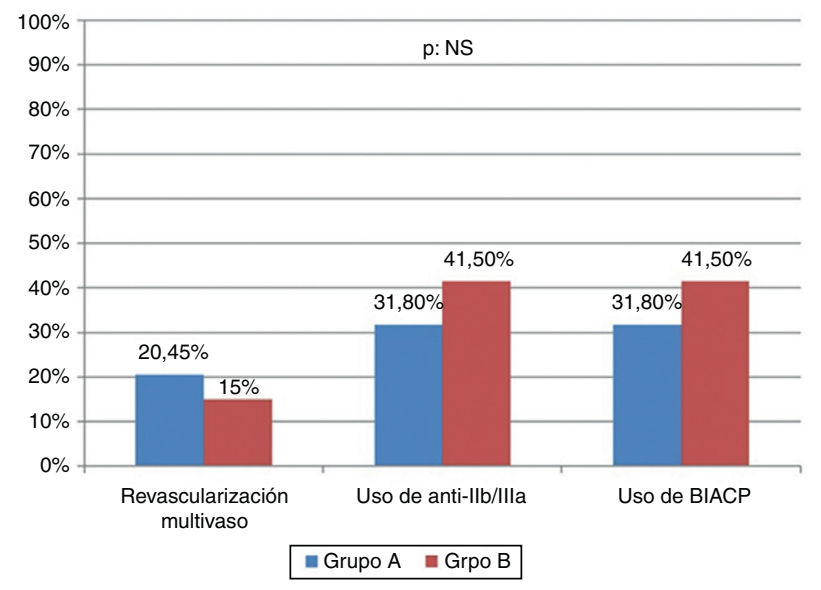

Figura 1 Revascularización multivaso, uso de anti-Ilb/Illa y BIACP. BIACP: balón intraaórtico de contrapulsación. 


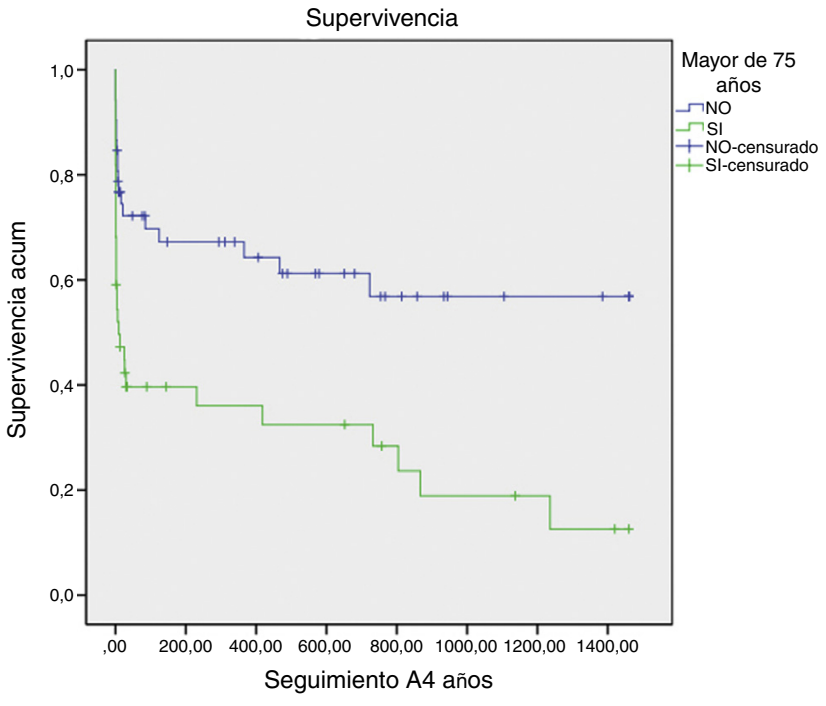

Figura 2 Supervivencia durante el seguimiento a 4 años $(p=0.0001)$.

supervivencia fue de 30 días (rango intercuartílico de 2-651 días). Al evaluar el objetivo principal de nuestro estudio y comparar la mortalidad entre ambos grupos al final del seguimiento observamos que, a pesar de la estrategia invasiva en cuanto a intervencionismo coronario, en el grupo de pacientes mayores de 75 años (grupo A) el objetivo principal fue significativamente superior ( 32 pacientes fallecidos, 73\%) al de los pacientes menores de 75 años (grupo B, 20 pacientes fallecidos, 38\%) $(p=0.002)$.

La tasa de incidencia del global de la muestra (incidence rate), como marcador del ritmo de ocurrencia del objetivo principal, fue de 11.2 eventos por cada 100 pacientes y año de seguimiento.

La curva de Kaplan-Meier (fig. 2) muestra una menor supervivencia en el seguimiento en los pacientes mayores de 75 años respecto a los pacientes menores de 75 años (Long Rank $13.16 \mathrm{p}=0.0001$ ).

Como se puede observar en la gráfica, a pesar de una disminución mantenida a largo plazo en la supervivencia de los pacientes mayores de 75 años, la mayor diferencia entre ambos grupos se produce durante el ingreso hospitalario (variable secundaria), apareciendo durante el mismo un total de 24 fallecimientos (54.5\%) en el grupo A y 16 fallecimientos $(30.2 \%)$ en el grupo B $(p=0.02)$. Sin embargo, a pesar de que la mayor diferencia se observa durante el ingreso hospitalario, como se puede observar en la figura 3 la supervivencia durante el seguimiento desde el alta hospitalaria es significativamente superior en los pacientes del grupo $B$ frente a los pacientes mayores de 75 años $(p=0.016)$.

\section{Discusión}

El choque cardiogénico es la principal causa de muerte hospitalaria de los pacientes que sufren un IAM y afecta al 5-8\% de los pacientes que ingresan en el hospital con ese diagnóstico. Su incidencia no ha sufrido grandes modificaciones en los últimos años, desarrollándose más frecuentemente en pacientes de edad avanzada, mujeres, diabéticos, pacientes

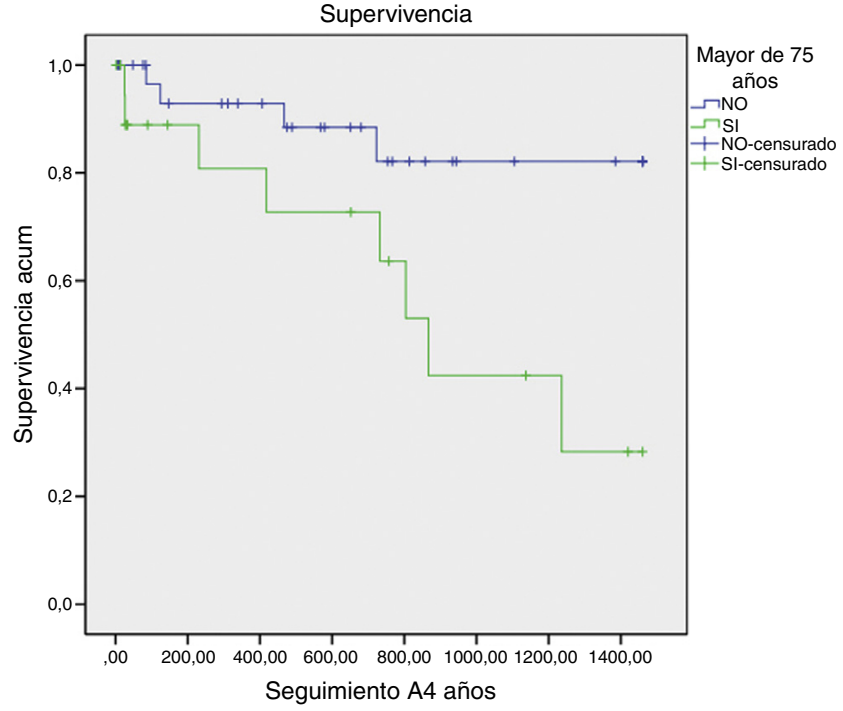

Figura 3 Supervivencia desde el alta hospitalaria $(p=0.016)$.

con infarto previo y con infartos extensos que afectan más del $40 \%$ de la masa ventricular izquierda. Se ha demostrado que los pacientes de edad avanzada que sufren un choque cardiogénico de origen isquémico tienen un peor pronóstico hospitalario y en el seguimiento, sin embargo hay pocos estudios que evalúen el pronóstico a largo plazo de este grupo de pacientes, dada la selección no aleatoria de pacientes para un tratamiento intensivo lo que supone un sesgo que puede incrementar el beneficio real de esta intervención, ya que se actúa de forma invasiva en los pacientes con más alta probabilidad de sobrevivir (jóvenes, sin infarto previo, sin enfermedades concurrentes).

En el estudio PRIAMHO ${ }^{10,11}$ llevado a cabo en hospitales españoles, en los pacientes mayores de 75 años con IAM la trombólisis se asociaba con un curso evolutivo menos complicado y con una menor mortalidad a 28 días (el 27 frente al $31.3 \%$ del grupo con tratamiento convencional; $p=0.035$ ). Sin embargo, estos resultados estaban mediados en parte por otras variables como la edad, uso más frecuente de ácido acetilsalicílico y un mayor número de pacientes en clase Killip III-IV excluidos de la terapia trombolítica.

En el año 2005, tras la realización de una encuesta en hospitales españoles con capacidad para realizar angioplastias primarias, se descubrió que el $42 \%$ de los mayores de 75 años ingresados por IAM no recibían tratamiento alguno de reperfusión, y de los que lo recibían, la mayoría eran tratados con fibrinólisis en vez de con angioplastia primaria.

Por todas estas razones, las secciones de Cardiopatía Isquémica y Cardiología Intervencionista de la Sociedad Española de Cardiología, impulsaron el estudio TRIANA ${ }^{12}$ (TRatamiento del Infarto Agudo de miocardio en Ancianos) con el objetivo de comparar ambas estrategias y descubrir cuál resulta más efectiva en este subgrupo de pacientes, cada vez más amplio.

El estudio se realizó en 23 hospitales españoles entre marzo de 2005 y diciembre de 2007. Se analizaron pacientes mayores de 75 años que acudieron a las unidades coronarias con IAMEST de menos de $6 \mathrm{~h}$ de evolución, y que eran candidatos óptimos para la fibrinólisis. 
Estos 266 pacientes fueron aleatorizados con angioplastia primaria y fibrinólisis, y se les realizó un seguimiento de 12 meses, descubriendo así que, en este caso, la angioplastia primaria lograba un $6.5 \%$ menos de muertes, reinfartos o accidentes cerebrovasculares incapacitantes en mayores de 75 años. Concretamente, el tratamiento de la angioplastía primaria se asoció con un $18.9 \%$ de aparición de estos eventos en los 30 días siguientes a la intervención, mientras que con la fibrinólisis se produjeron un $25.4 \%$ de estos episodios.

Tras combinar los resultados de este estudio con los 2 ensayos anteriores de reperfusión en pacientes mayores de 75 años, se comprobó definitivamente una superioridad de la angioplastia primaria frente a la fibrinólisis en reducir resultados desfavorables como la muerte, nuevo infarto o ictus.

Pese a la clara superioridad del intervencionismo en la población anciana, hemos de tener en cuenta que en este estudio no se incluyeron pacientes en situación de choque cardiogénico.

Si realizamos una revisión de los escasos estudios publicados, el mayor registro publicado hasta la fecha sobre choque cardiogénico de origen isquémico es el SHOCK Trial ${ }^{13,14}$. Los resultados iniciales de este estudio demostraron que los pacientes con choque cardiogénico de origen isquémico tratados mediante revascularización percutánea precoz tenían una mayor supervivencia a los 30 días, 6 meses y al año que los pacientes que recibieron tratamiento médico convencional, no siendo refutados estos hallazgos en el grupo de pacientes mayores de 75 años. En cuanto a mortalidad entre pacientes tratados mediante revascularización percutánea precoz, este estudio demuestra el peor pronóstico de los pacientes mayores de 75 años (mortalidad hospitalaria $75 \%$ y al año $79.2 \%$ ) frente al grupo de pacientes más jóvenes (mortalidad hospitalaria $41.4 \%$ y al año $48.4 \%$.), hallazgo concordante con los resultados de nuestro trabajo. Sin embargo, un subanálisis posterior ${ }^{15}$ demostró que tanto en los pacientes mayores como en los menores de 75 años existía un beneficio estadísticamente significativo en cuanto a supervivencia a 30 días en los pacientes que recibieron revascularización percutánea en las primeras $18 \mathrm{~h}$ del inicio del cuadro frente a los pacientes que fueron revascularizados más tardíamente o se manejaron de manera conservadora. En cuanto a mortalidad hospitalaria entre ambos grupos de este subanálisis, no existieron claras diferencias. En el año 2006 Migliorini et al. ${ }^{16}$ publicaron un estudio en el que se demuestra la clara influencia de la edad en cuanto a mortalidad a los 6 meses de 104 pacientes mayores de 75 años con diagnóstico de choque cardiogénico de origen isquémico que recibieron revascularización percutánea en las primeras $6 \mathrm{~h}$ del desarrollo del cuadro frente a pacientes menores de 75 años. La mortalidad de los pacientes mayores fue significativamente más elevada (56\%) que la de los pacientes menores de 75 años (26\%).

Otro estudio publicado en 2008 por Guo et al. ${ }^{17}$ también demostró el beneficio de la revascularización percutánea precoz en pacientes mayores de 75 años con choque cardiogénico secundario a un IAM frente a los pacientes que recibieron tratamiento médico.

A pesar de los datos anteriores, Lim et al. ${ }^{18}$ publicaron en 2009 los datos de un estudio realizado en pacientes con IAM complicado con choque cardiogénico que recibieron revascularización coronaria percutánea precoz, y compararon la supervivencia hospitalaria, a los 30 días y al año entre pacientes mayores y menores de 75 años. Los resultados no mostraron diferencia significativa en cuanto a mortalidad hospitalaria, a los 30 días y al año de seguimiento entre ambos grupos. Estos resultados contrastan con la evidencia anteriormente expuesta de la influencia de la edad en el peor pronóstico de estos pacientes. Nuestro estudio, no aleatorizado, describe los resultados clínicos y angiográficos del intervencionismo coronario electivo en pacientes con choque cardiogénico secundario a un IAM.

En él se analiza a este subgrupo de pacientes añosos, demostrando que a pesar de recibir un tratamiento intensivo mediante revascularización coronaria percutánea, los pacientes mayores de 75 años tienen mayor mortalidad durante un seguimiento máximo de 4 años (fallecen el 73\%) en comparación con el grupo de pacientes más jóvenes, que presentan una mortalidad del $38 \%$ al final del seguimiento. Hay que destacar que a pesar de mantenerse constante esta diferencia durante el seguimiento, es durante el ingreso hospitalario cuando se produce la mayor diferencia de mortalidad, ya que más del $50 \%$ de los pacientes mayores de 75 años fallecen en este periodo en comparación con el $30 \%$ de los pacientes más jóvenes. Vemos cómo la edad influye drásticamente en la supervivencia de este grupo de enfermos a pesar de que en el grupo de pacientes más jóvenes había un mayor número de pacientes diabéticos y fumadores, 2 factores de riesgo cardiovascular claramente relacionados con el aumento de la mortalidad en pacientes que sufren un infarto de miocardio. Hemos de considerar el beneficio neto de la angioplastia primaria en pacientes de mayor riesgo cardiovascular como son los mayores de 75 años frente a la trombólisis o manejo conservador, pese a las elevadas cifras de mortalidad en este contexto clínico.

\section{Implicaciones clínicas}

1 El choque cardiogénico sigue siendo una patología con elevada mortalidad a corto plazo pese a la utilización de la estrategia invasiva inicial y de tratamiento médico óptimo.

2 Entre los pacientes en choque cardiogénico y angioplastia primaria de nuestra muestra, aquellos de mayor edad presentan más eventos adversos, con una mortalidad del $50 \%$ durante el ingreso, aunque el beneficio clínico en estos pacientes probablemente es superior, dado el mal pronóstico a corto plazo del choque cardiogénico.

\section{Limitaciones}

No se realizó una evaluación funcional de los pacientes mayores de 75 años. Puede que la diferencia de supervivencia fuese menor entre pacientes de ambos grupos que presentasen una capacidad funcional similar.

\section{Conclusión}

El choque cardiogénico de origen isquémico en pacientes mayores de 75 años presenta una alta mortalidad durante el ingreso hospitalario que continúa empeorando de forma significativa en el seguimiento a largo plazo. Podríamos 
plantearnos seleccionar a qué pacientes de estas características ofrecemos tratamientos intensivos e implementar seguimientos clínicos más cercanos y estrechos.

\section{Conflicto de intereses}

Los autores declaran no tener ningún conflicto de intereses.

\section{Bibliografía}

1. Rogers WJ, Canto JG, Lambrew CT, et al. Temporal trends in the treatment of over 1.5 million patients with myocardial infarction in the US from 1990 through 1999: The National Registry of Myocardial Infarction 1, 2 and 3. J Am Coll Cardiol. 2000;36:2056-63.

2. Kesteloot H, Sans S, Kromhout D. Evolution of all-causes and cardiovascular mortality in the age-group 75-84 years in Europe during the period 1970-1996. A comparison with worldwide changes. Eur Heart J. 2002;23:384-98.

3. Marrugat J, Elosua R, Martí H. Epidemiología de la cardiopatía isquémica en España: estimación del número de casos y de las tendencias entre 1997 y 2005. Rev Esp Cardiol. 2002;55:337-46.

4. Floyd KC, Yarzebski J, Spencer FA, et al. A 30-year perspective (1975-2005) into the changing landscape of patients hospitalized with initial acute myocardial infarction: Worcester Heart Attack Study. Circ Cardiovasc Qual Outcomes. 2009;2:88-95.

5. Pérez-Castellano N, García E, Serrano JA, et al. Efficacy of invasive strategy for the management of acute myocardial infarction complicated by cardiogenic shock. Am J Cardiol. 1999;83:989-93.

6. Chuquiure E. Consideraciones fisiopatológicas actuales del choque cardiogénico asociado a los síndromes isquémicos coronarios agudos. Arch Cardiol Mex. 2006;76 Supl 2:258-60.

7. Lupi E, González H. Choque cardiogénico por síndrome isquémico coronario agudo sin complicaciones mecánicas. Arch Cardiol Mex. 2007;77 Supl 1:34-8.

8. Hochman JS, Sleeper LA, Webb JG, et al. Early revascularization in acute myocardial infarction complicated by cardiogenic shock. SHOCK Investigators. Should We Emergently Revascularize Occluded Coronaries for Cardiogenic Shock. N Engl J Med. 1999;341:625-34.
9. Elandt-Johonson RC, Johnson NL. Survival models and data analysis. New York: John Wiley \& Sons; 1980. p. 14-6.

10. Arós F, Cuñat J, Loma-Osorio A, et al. Management of myocardial infarction in Spain in the year 2000. The PRIAMHO II study. Rev Esp Cardiol. 2003;56:1165-73.

11. Pabón P, Fernando B, San José-Garagarza JM, et al. Trombolisis en el anciano con infarto agudo de miocardio. Estudio PRIAMHO. Rev Esp Cardiol. 2000;53:1443-52.

12. Bueno H, Betriu A, Heras M, et al. Primary angioplasty vs. fibrinolysis in very old patients with acute myocardial infarction: TRIANA (Tratamiento del Infarto Agudo de miocardio eN Ancianos) randomized trial and pooled analysis with previous studies. Eur Heart J. 2011;32:51-60.

13. Hochman JS, Buller CE, Sleeper LA, et al. Cardiogenic shock complicating acute myocardial infarction-etiologies, management and outcome: a report from the SHOCK Trial Registry. Should we emergently revascularize occluded coronaries for cardiogenic shock? J Am Coll Cardiol. 2000;36:1063-70.

14. Dzavik V, Sleeper LA, Picard MH, et al., SHould we emergently revascularize Occluded Coronaries in cardiogenic shock Investigators. Outcome of patients aged $>$ or $=75$ years in the SHould we emergently revascularize Occluded Coronaries in cardiogenic shock (SHOCK) trial: Do elderly patients with acute myocardial infarction complicated by cardiogenic shock respond differently to emergent revascularization? Am Heart J. 2005;149:1128-34.

15. Dzavik V, Sleeper LA, Cocke TP, et al. Early revascularization is associated with improved survival in elderly patients with acute myocardial infarction complicated by cardiogenic shock: a report from the SHOCK Trial Registry. Eur Heart J. 2003;24:828-37.

16. Migliorini A, Moschi G, Valenti R, et al. Routine percutaneous coronary intervention in elderly patients with cardiogenic shock complicating acute myocardial infarction. Am Heart J. 2006;152:903-8.

17. Guo L, Mai X, Deng J, et al. Early percutaneous intervention improves survival in elderly patients with acute myocardial infarction complicated by cardiogenic shock. Kardiol Pol. 2008;66:722-6.

18. S. Lim H, Farouque O, Andrianopoulos N, et al., Melbourne Interventional Group. Survival of elderly patients undergoing percutaneous coronary intervention for acute myocardial infarction complicated by cardiogenic shock. JACC Cardiovasc Interv. 2009;2:146-52. 